\title{
Refractory Acute Lymphoblastic Leukemia
}

National Cancer Institute

\section{Source}

National Cancer Institute. Refractory Acute Lymphoblastic Leukemia. NCI Thesaurus.

Code $C 136488$.

Acute lymphoblastic leukemia that does not respond to treatment. 